\title{
Health in the palm of your hand-part 1: the risks from smartphone abuse and the role of telemedicine and e-Health
}

\author{
Daniele Giansanti, Giovanni Maccioni \\ Centro TISP, Istituto Superiore di Sanità, Roma, Italy \\ Contributions: (I) Conception and design: All authors; (II) Administrative support: None; (III) Provision of study materials or patients: All authors; (IV) \\ Collection and assembly of data: D Giansanti; (V) Data analysis and interpretation: D Giansanti; (VI) Manuscript writing: All authors; (VII) Final \\ approval of manuscript: All authors. \\ Correspondence to: Daniele Giansanti. Centro TISP, Istituto Superiore di Sanità, via Regina Elena 299, 00131, Roma, Italy. \\ Email: daniele.giansanti@iss.it; gianslele@gmail.com.
}

\begin{abstract}
Background: The evolution of the Telemedicine and e-Health is strictly related to the evolution of the mobile-technology. If today it is possible to successfully use a wide range of mHealth applications, playing an important role in Telemedicine and e-Health, health care has surely a gratitude debt with the smartphone. However, it is increasingly becoming clear that excessive use of the smartphone has implications for health in terms of risks and it is therefore necessary to tackle the various gaps and/or the role that Telemedicine and e-Health itself has in this field.

Methods: Through an overview to literature, Web and App stores the study faced the implications for health from the excessive use of the smartphone and therefore the various gaps and/or the role that Telemedicine and e-Health itself has in this area both in the prevention and in the monitoring with particular regard to the three components: eHealth, mHealth and eLearning.

Results: The study found (I) as emerging risks: the text neck, the risk of addiction and the risk in the use of deceptive Apps in mHealth; (II) as Telemedicine and e-Health useful approach: the design of Apps for the text neck prevention and the relevant acceptance and effectiveness assessment; the design and application of electronic surveys for tele-assessment of the addiction risk; the design and application of information and training path on the deceptive Apps in mHealth.

Conclusions: The key role of Telemedicine and e-Health in the prevention and monitoring of the risks from smartphone abuse has been clearly identified and the young people has been identified as target population and the relevant need to design specific teaching packages in this field focused on them.
\end{abstract}

Keywords: Mobile-health; smartphone; text neck; addiction; telemedicine

Received: 17 June 2020; Accepted: 06 December 2020; Published: 20 July 2021.

doi: $10.21037 /$ mhealth-20-150

View this article at: http://dx.doi.org/10.21037/mhealth-20-150

\section{Introduction}

The evolution of mobile-health is strictly related to the evolution of the mobile technology. If today it is possible to successfully use a wide range of mobile applications, playing an important role in Telemedicine and e-Health $(1,2)$, we have surely a gratitude debt with the smartphone, which, as it is well known is an evolution of the cell-phone. However, it is increasingly becoming clear that excessive use of the smartphone has implications for health in terms of risks and it is therefore necessary to tackle the various gaps and/ or the role that Telemedicine and e-Health itself has in this field in the prevention and in the monitoring with particular regard to the three components: e-bealth, $m$-bealth and e-learning. It is furthermore important the information and the training in this field and therefore to design and apply new teaching packages/modules, which face both the abuse risks and the contribution of Telemedicine and e-Health. 
The global study is arranged into two companion papers structured as commentaries.

(I) The first paper "Part 1: the risks from smartphone abuse and the role of telemedicine and e-Health" here presented addresses the risks from smartphone abuse and tackle the various gaps and/or the role that Telemedicine and e-Health itself has in this field.

(II) The second paper "Health in the palm of your hand. Part 2: design and application of an educational module for young people on the risks from smartphone abuse and the opportunities of telemedicine and e-Health" (3) deals with the design and application of a new teaching package/module dedicated to young people, the target population, which faces both the abuse risks and the approach to the problem also based on several Telemedicine and e-Health components.

\section{From the phone cell to the smartphone}

In general, the smartphone as we know it today differs from the mobile phone due to the simultaneous presence of the following features:

(I) The increased memory, a superior computing capacity, a much more advanced data connection capacity due to the presence of dedicated operating systems;

(II) A great potential for production and management of multimedia content such as taking high resolution photos, producing video clips;

(III) The ability to easily install features and/or applications (Apps);

(IV) The equipment of a high resolution touch screen;

(V) The ability to use/maneuver a virtual keyboard to interact with the different features of the device (from the phonebook to the notepad), with the web, with the various applications installed and with so-called social networks;

(VI) Integration with sensors such as accelerometers, gyroscopes, magnetometers, thermometers etc.;

(VII) The possibility of wireless tethering, WiFi or Bluetooth, with other devices such as other smartphones or cell phones, laptops or fixed computers.

It is precisely the possibility of interacting in a userfriendly way with the device through the virtual keyboard which, as will be seen below, contributes significantly to the appearance of new risks. In fact, Who of us do not remember the complexity of sending a message with a mobile phone via the Short Message Service (SMS) system using the same call composer? This complexity combined with the unit cost of the message limited the use of the mobile phone in the socalled texting activity.

Currently, smartphones can be configured with numerous free or paid downloadable Apps by accessing "virtual stores". Some examples of these virtual stores are the Apple App Store, the Android Play Store, the Windows Store for Windows Phone and Windows 10 Mobile, Blackberry World for Blackberry OS/Blackberry 10 and the Ovi Store for Symbian OS. The first mobile devices, with the touch screens are the 2006 Prada Phone from LG, the Apple iPhone from 2007 and the 2008 HTC T-Mobile G1 which is also the first smartphone with the Android operating system, the most widespread in the world and adopted by most manufacturers. Starting from these years therefore, smartphones have been spreading as we know them today with the features listed above.

\section{Between opportunities and problems}

The important developments in the Information and Communication Technology (ICT) in the last decade have made applications possible of smartphones and tablets in industry, in the consumer sector and in healthcare with the mobile-health. The smartphone is certainly the most used among mobile technologies. In the USA, $87 \%$ of teenagers (between the ages of 14 and 18) own a smartphone and $79 \%$ of teenagers (aged 12-15) in Great Britain (4). As a multifunctional device, the smartphone allows access to the network for multimedia entertainment, to communicate via chat and social networks, for video calls, for downloading of contents and applications, and finally for reading textual contents such as books. The flip side of the coin is highlighted by placing attention to the time of use and the type of physical activity resulting from the interaction between subject and smartphone, or the resulting motor/ postural task interaction. As highlighted in the review of the literature by Toh et al. (4) is being recorded in particular a growing increase in time dedicated to smartphone in nonvoice activities, which has rapidly grown and is around 3 hours a day (US data); this means that you can spend up to 1,095 hours/year to read and write with the smartphone. An exposition of this entity appears relevant for the risk to exposure. 


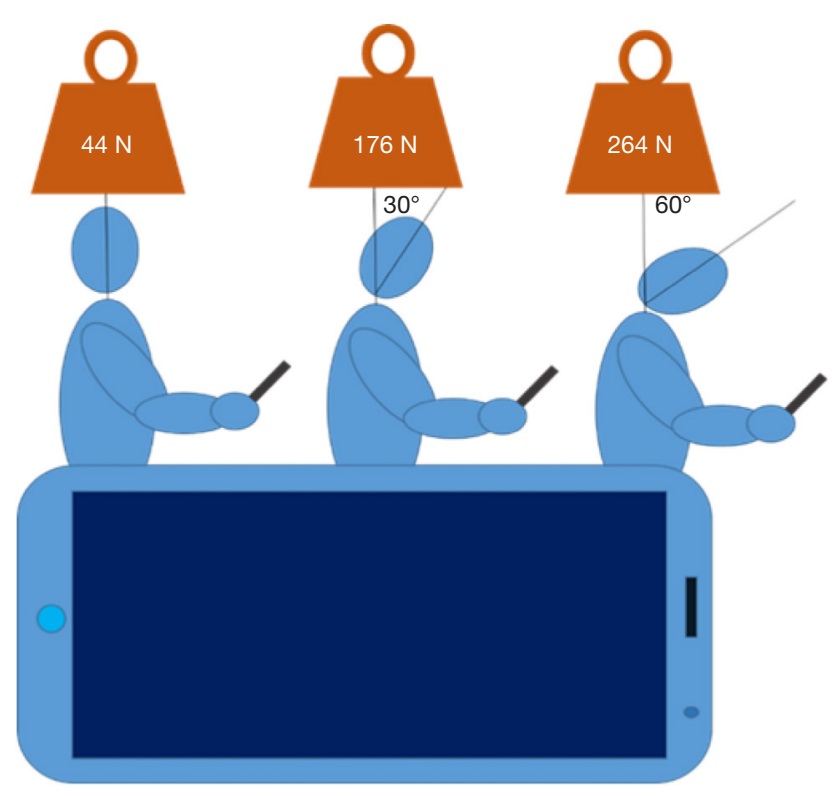

Figure 1 Relationship between angle tilt and load.

\section{Emerging risks from technology abuse}

The resulting motor-postural task while using smartphones is particularly important considering that the user can spend two to four hours a day with this device to read or write, in all between 700 and 1400 hours a year! The text neck $(4,5)$ is a definition used in recent years to describe stress injuries repeated and neck pain resulting (I) from an incorrect interaction with the devices (II) used for an excessive period of time (6). The text neck can be considered a neuromusculoskeletal risk from abuse of smartphone technology (6-11). The ease of interaction and the enormous potential of the smartphone device can generate the so-called psychocognitive risk of addiction. Particular object of study are the methodologies for assessing these new forms of addiction risk in adolescents and in the young adults through the administration of psychocognitive tests that are recently the subject of research and transfer in the various languages (12-17). When using the smartphone particularly important is the misuse of Apps that can be confused with medical Apps even though they are not. Think of the Apps for the Wellness/Fitness or Apps that provide non-certified parameters for medical use. With the introduction of the smartphones we must also face these risks i.e., the risks from incorrect use of certain types of applications. Other risks inherited from mobile phones are:

(I) Risk for vision, e.g., from fatigue or from blue light with high energy;
(II) Electromagnetic risk;

(III) Risk to hearing.

These risks are still the subject of studies and experimental research activities dedicated both to the quantification of the risk and to the comparison between the two cellular and smartphone technologies. Prevention solutions for these inherited risks have been identified since the cell-phone age and consists on protective screen for the risks for the vision and specific headphones for the other ones.

\section{Methods}

The aims of this study is to face the implications for health from the excessive use of the smartphone and therefore the various gaps and/or the role that Telemedicine and e-Health itself bas in this area both in the prevention and in the monitoring with particular regard to the three components: $e$-health, $m$-health and e-learning.

In consideration to this the methodological flow was based on three polarities for each one of the three considered topics (text neck, risk of addiction, smartphone abuse, smartphone incorrect use) faced in this study:

(I) A careful analysis of the state of art and a deep overview of the relevant connected implication/ problems;

(II) An overview of the technological implications/need with a careful consideration on mHealth being the focus of the journal;

(III) An overview of the possible needs in the fields highlighting and discussing the possible implications, from the training up to the involved actors.

\section{Results}

\section{The text neck}

\section{The syndrome}

The text neck is a term recently used to describe postural problems coming from an incorrect postural interaction with the smartphone device (6). It has recently replaced the term turtle neck (6). It has been demonstrated that the text neck is manly caused by an incorrect inclination angle (Figure 1) (6,7). Some studies based on informatics based on mathematic solid modelling highlighted that the incorrect neck angles strongly increase the load at the cervical vertebral level (9), with 60 degrees of inclination the load 


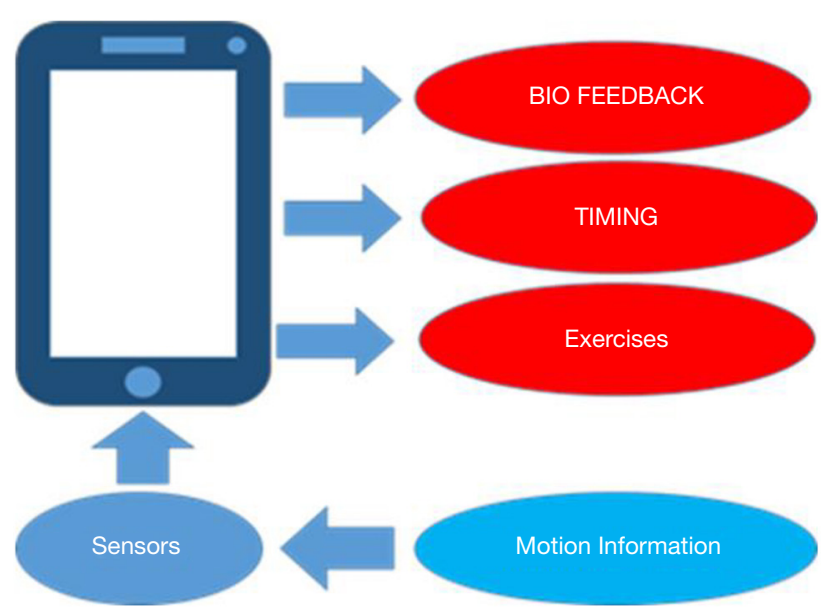

Figure 2 The smartphone and the functionalities for the text neck prevention.

can reach $264 \mathrm{~N}$ ! Principally this syndrome is affecting the mobile born, i.e., the very young subjects $(8-10)$. This is a very important problem as these subjects have not yet completed their muscle/bone formation!

Among the medical problems we can found

(I) Continuous headaches;

(II) Cervical problems;

(III) Rigidity in the region between the two arms and the cervical area.

Scientists (5), suggest simple approaches. It is, for example recommended to use the smartphone in a vertical orientation at the eye level and use both the two arms and hands in an ergonomic way (for example maintaining a symmetrical arm posture).

\section{The role of the Telemedicine and e-Health The development of m-bealth solutions}

There are numerous wearable biomedical instruments (18-20) used for motion analysis able to record the activity and at the same time give feedback to the user. However, a solution can come from mobile technologies. In fact, these systems, use for example, movement sensors such as the accelerometers, used in the Apps (as for the smartphones) for the motion activity monitoring or as an inclinometer. The smartphone therefore can be useful to protect us from the TN, aiding us to put the device at the level of the eyes. Figure 2 first illustrates how the smartphone thanks to the accelerometer (used as an inclinometer) can return a biofeedback information to the user related to the inclination (biofeedback). An alignment with respect to the terrestrial vertical pushes the user to take the device to eye level and to assume a correct posture. Instead an inclination with respect to the terrestrial vertical forces the user to bend the neck and assume one incorrect posture by increasing the load on the cervical vertebrae. This "virtual level function" can be mapped with a visual, sound or vibrotactile biofeedback, using the actuators of the smartphone itself. A symbol with a red color, can, for example, indicate an inclination associated with incorrect device and therefore posture, a green symbol can indicate a correct position of the device (enforcing a correct posture), other colors can be used to indicate with gradation several device inclinations that lead to incorrect postures. Figure 2 also show other potentialities of the smartphone. In particular, using internal registers it is possible to be updated on the timing of use. Linked to these features, other functions may suggest physical exercises (which depend on the time of use), as recommended by postural scholars in this field. We carried out a specific search on the Android Google Play Store, dedicated to Apps that take advantage of the principle described in Figure 2. Some not exhaustive examples of these Apps are: Text Neck indicator; HeadUp—Protect your neck!; Text Neck. For example the App HeadUp—protect your neck! that we have tried with the android operative system returns a biofeedback on the inclination of the device based on a circle that changes color based on the angle (Figure 3).

\section{The issues to deepen in Telemedicine and e-Health}

It is clear that the availability of Apps like the ones mentioned above can represent an opportunity for users of mobile technology: "the same mobile technology protects me from mobile technology" through the tools of selftherapy and self-empowerment, furthermore data are stored in a cloud and user can consult them daily as a diary/log. At this point, however, the contribution of Telemedicine and e-Health is in two directions:

(I) It is necessary to put in place procedures that allow for the assessment of: (i) the acceptance of these Apps (by the actors concerned, especially young people and medical and physiotherapist specialists) and (ii) the effectiveness;

(II) It is necessary to disseminate among young subjects the prevention solutions on the TN based on the suggestions of the experts and on m-health.

\section{The addiction risk}

The psychological point of view in brief

The ease of interaction and the enormous potential of 

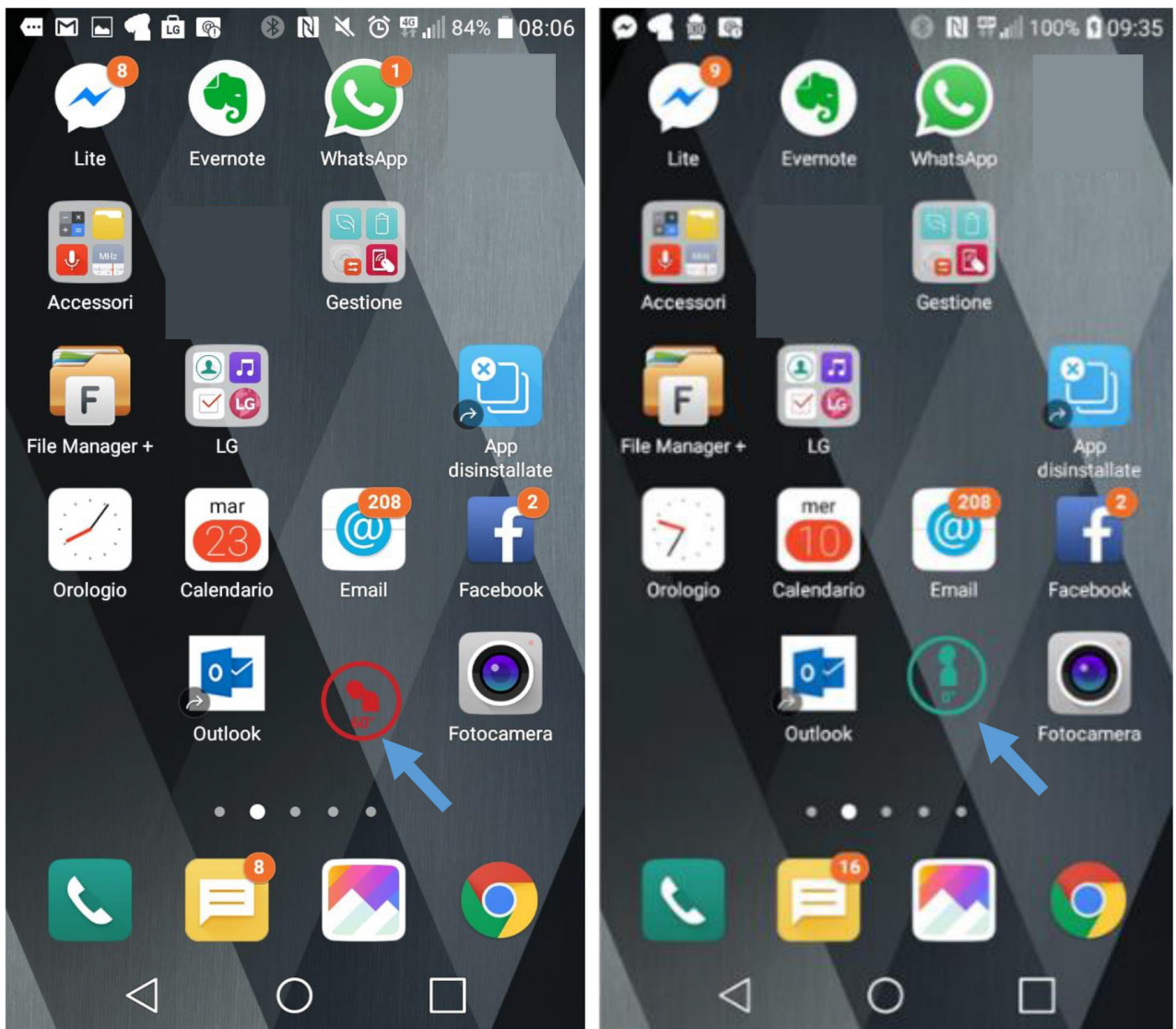

Figure 3 The biofeedback used in an App: red means a device inclination of 60 degs; green means an inclination of 0 degs.

the smartphone device have intensified the psychologicalcognitive risks (12-14). For some authors the addiction risk (12) in the smartphone is the source for the other psychological problems. Particular object of study in this field are the methods of risk assessment in the adolescents and young adults through the administration of psychological and cognitive questionnaires that have recently been the subject of research and transfer in various languages (15-17).

\section{The role of the Telemedicine and e-Health}

The role of Telemedicine and e-Health is to focus on psychological test methodology and to set-up surveys through an electronic based procedure using the cyberpsychology approach familiar in the tele-psychology applications. In the whole study considering the two parts, we found useful one of the tests used in this field: the Smartphone Addiction Scale Short Version (SAS-SV) test, a psychological test based on a specific questionnaire that is self-administered and used to identify the risk level of smartphone addiction and that allows categorization in various risk groups in adolescents and young adults. We report here this test that was used in part 2 of this study (3). This test was validated on a large sample (15) and has since been validated in other languages $(16,17)$. In Italy it has been recently validated (5) in the national idiom. The test includes 10 statements related with the interaction with the smartphone.

To these statements each subject must assign a graded assessment at 6 levels; the first level corresponds to the lowest rating, i.e., the minimum assignable score (strongly disagree); while level 6 corresponds to the highest rating, i.e., the maximum score assignable (strongly in agreement). The scores obtained on each individual question are then added together. The value obtained identifies belonging to the risk class. The risk classes identified by the test are different based to sex. Males are considered technology dependent with a score above 31 , with high risk of dependence with a score between 22 and 31 . Females are considered technology-dependent with a score higher than 33 , with 


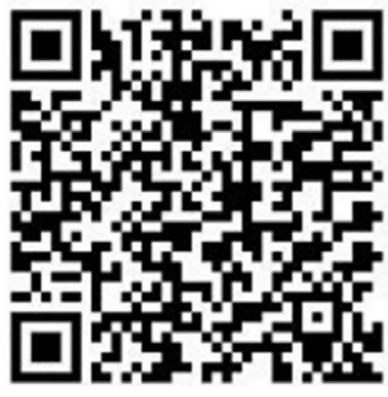

https://onedrive.live.com/survey?resid=AE230E99800FB7C8!124642\&authke $\mathrm{y}=$ !AHS_RHjijee29Aw

Figure 4 The electronic survey developed using Onedrive: QR code and internet pointer.

high risk of addiction with scores between 22 and 33 .

There are several solutions to develop this survey electronically. For example it is possible to open a Microsoft account; use Onedrive (Microsoft, USA), design the survey and to send it using the messengers or the emails. The answers are centralized on excel online in the account. Figure 4 shows the internet link and the QR-code relevant to an SAS-SV survey in Italian designed using Onedrive.

\section{The risk in the use of deceptive APPs in Mobile-bealth}

To face this risk is necessary to pay attention to those Apps (21-23) in relation to which the citizen can be confused with respect to the intended use. There are in particular App categories not for medical use where the borderline with medical Apps really is thin. Among other things, all these Apps listed here seem to behave just like the the Apps for Digital Health: they record the parameters, save them in a cloud, create a sort of virtual diary that can be consulted via a mobile navigation everywhere.

The case of the App for wellness and fitness

Consider, for example, the numerous Apps developed for fitness and wellness. It is sufficient to browse the web and check how the proliferation of this type of Apps is particularly marked, ranging from the Apps for tracking the walk/run up to Apps that propose you a diet (considered by the proponents of the App) healthy. Along with the proliferation of fitness and wellness Apps, we are simultaneously witnessing the apparently uncontrolled proliferation of blog sites on this type of Apps and on many others. They are sites where supposed experts promote App groups, rather than others, sometimes with a mechanism not very dissimilar to that of "cook blogs", thus causing similar concerns. The central point however is that the citizen must pay special attention in the interpretation of the parameters provided by these Apps. In fact these Apps are not validated as Medical Devices. Another important aspect that must be considered is the spread of self-fitness and self-wellness; without resorting to the valuable advice of experienced professionals, for example in the field of physical activity/sports and nutrition. With the release of the recent App Sweatcoin that rewards you for walking with a virtual coin, there is, for example a rush to always do more physical activity to have more and more virtual money to spend, without the obligation of a medical. Health risks are evident!

\section{Apps that can be confused with medical ones}

The App-stores are now full of many Apps that can confuse (21-23) the citizen on the intended use. Examples that intrigue and worry at the same time are numerous. Just go to Play Store, to find Apps that promise to detect your blood pressure or the temperature or even the blood sugar with the simple support of the fingertip (Figure 5). It's obvious how the ordinary citizen, faced with these Apps, risks becoming disoriented. Only three examples have been brought, but it is enough to navigate on the virtual shops to realize how the problem is decidedly broad and certainly worthy of attention. Very few citizens know what a medical device is and the possibility that these Apps are used in different way from the destination of use cannot be certainly excluded only trusting in the capacity of discernment of the citizen.

\section{The role of Telemedicine and e-Health}

Mobile Apps for devices such as smartphones and tablets are already in the thousands. Many Apps available today are easily confused with medical Apps. Think of the Apps for fitness and wellness and, more generally, to Apps that, even though they look medical, do not have such a destination. Consider the importance of the metabolic consumption parameter that in these Apps is deeply approximated and on which instead many build diets and regimes of life! Not to mention blood pressure, glycemic and cardiological parameters obtained using these non-medical Appparameters. Everything is aggravated by the birth of blog sites that promote this or that App and a widespread selftherapy mania. What is important (in addition to a capillary regulatory action) taking into account that the major users are young people, is to initiate training and information 

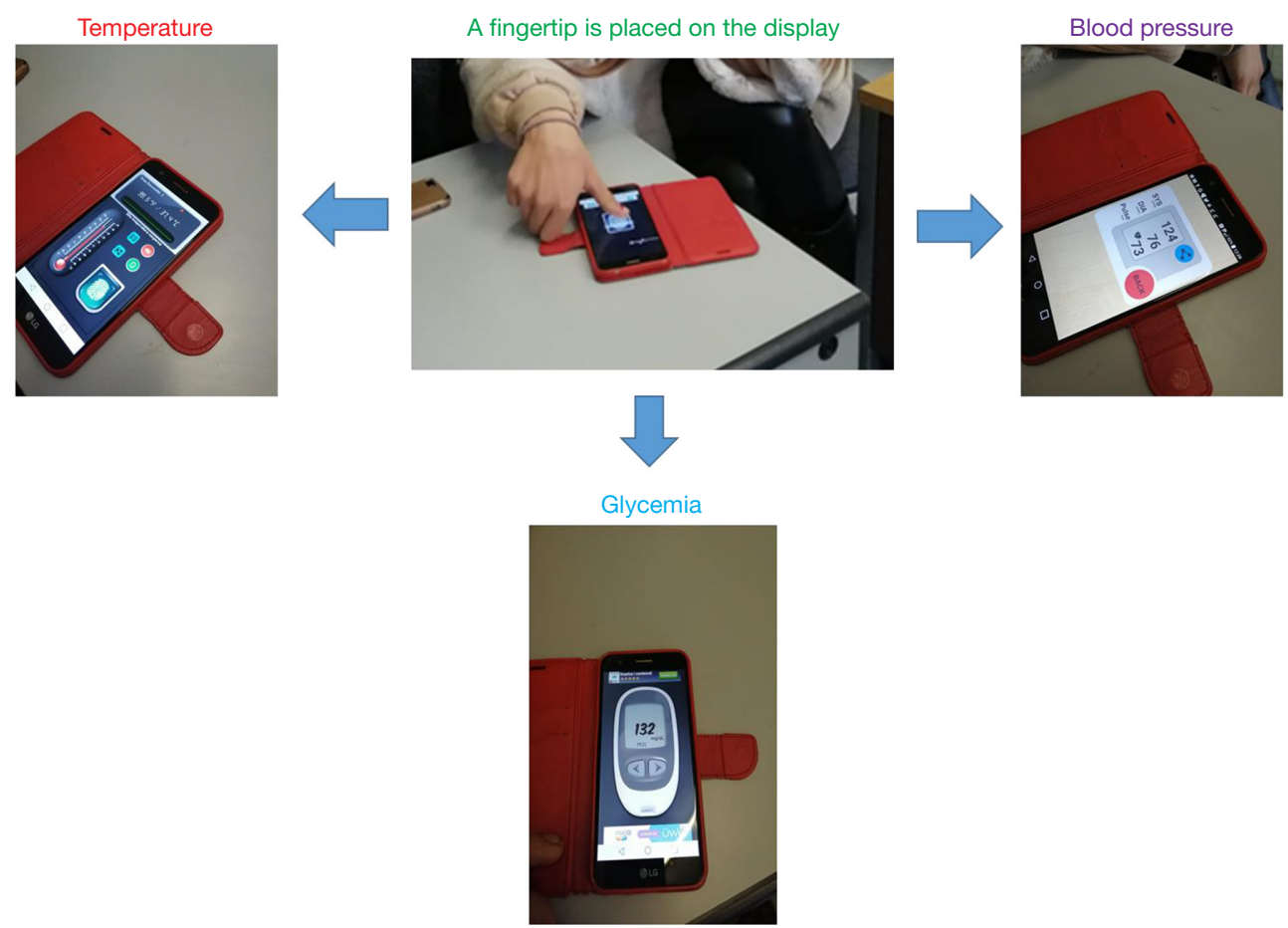

Figure 5 Example of Apps that with the typing of the fingertip appear as pressure meters; temperature meters; blood glucose meters.

courses on the correct use of these Apps from a young age also using e-learning tools and surveillance projects in the mainframe of their course of study and also through surveillance and monitoring initiatives conducted through dedicated electronic surveys.

\section{Conclusions}

The study here presented is a part of a global work arranged into two companion papers (3). The part here developed addresses the risks of smartphone abuse and tackle the various gaps and/or the role that Telemedicine and e-Health itself has in this field.

The faced risks from technology abuse were the following:

(I) The text neck (4-11) a postural neuromuscular risk (it is a definition used in recent years to describe stress injuries repeated and neck pain resulting (i) from an incorrect interaction with the devices (ii) used for an excessive period of time ).

(II) The addiction risk (12-17) with particular attention to the methodologies for assessing these new risks in adolescents and in the young adults through the administration of psychocognitive tests.
(III) The risk in the use of deceptive APPs in Mobilebealth (21-23). To face this risk is necessary to pay attention to those Apps in relation to which the citizen can be confused in use with respect to the intended use.

It was found that Telemedicine and e-Health can play a basic role.

For the text neck we enlightened the diffusion in mobilehealth of new Apps for the prevention of the text neck and the importance of the study on the acceptance and the effectiveness of these solutions and the information and training of young subjects.

For the addiction we enlightened how specific tests could be transformed in electronic surveys and used in an investigation using a telepsychology approach.

For the risk in the use of deceptive non-medical Apps we clarified that it was important (in addition to launch an adequate capillary regulatory action), taking into account that the major users are young people, to initiate (I) a training and information path on the correct use of these Apps, from a young age also using e-learning tools, surveillance projects in the mainframe of their course of study and also through (II) surveillance and monitoring initiatives conducted through dedicated electronic surveys. 
The outcome of this part was used as input in the companion paper (3) where it is reported the set-up and application of a specific teaching package/module in this field focused on young at the secondary school for a schoolwork alternation program according the Italian Law $107 / 2015(24,25)$.

\section{Acknowledgments}

Funding: None.

\section{Footnote}

Conflicts of Interest: Both authors have completed the ICMJE uniform disclosure form (available at http://dx.doi. org/10.21037/mhealth-20-150). DG serves as an unpaid editorial board member of $m$ Health from May 2019 to April 2021. The other authors have no conflicts of interest to declare.

Ethical Statement: The authors are accountable for all aspects of the work in ensuring that questions related to the accuracy or integrity of any part of the work are appropriately investigated and resolved. The ethical approval was not considered necessary, even the consent form was not necessary as the surveys were conducted anonymously.

Open Access Statement: This is an Open Access article distributed in accordance with the Creative Commons Attribution-NonCommercial-NoDerivs 4.0 International License (CC BY-NC-ND 4.0), which permits the noncommercial replication and distribution of the article with the strict proviso that no changes or edits are made and the original work is properly cited (including links to both the formal publication through the relevant DOI and the license). See: https://creativecommons.org/licenses/by-nc-nd/4.0/.

\section{References}

1. de la Torre-Díez I, López-Coronado M, Vaca C, et al. Cost-utility and cost-effectiveness studies of telemedicine, electronic, and mobile health systems in the literature: a systematic review. Telemed J E Health 2015;21:81-5.

2. Williams V, Kovarik C. WhatsApp: An Innovative Tool for Dermatology Care in Limited Resource Settings. Telemed J E Health 2018;24:464-8.

3. Giansanti D, Maccioni G. Health in the palm of your hand — part 2: design and application of an educational module for young people on the risks from smartphone abuse and the opportunities of telemedicine and e-Health. mHealth 2021;7:48.

4. Toh SH, Coenen P, Howie EK, et al. The associations of mobile touch screen device use with musculoskeletal symptoms and exposures: a systematic review. PLoS One 2017;12:e0181220.

5. Neupane S, Ifthikar Ali UT, Mathew A. Text-Neck syndrome - systemic review. Imperial J Interdisciplinary Res 2017;3:141-8.

6. Lee S, Kang H, Shin G. Head flexion angle while using a smartphone. Ergonomics 2015;58:220-6.

7. Hansraj KK. Assessment of stresses in the cervical spine caused by posture and position of the head. Surg Technol Int 2014;25:277-9.

8. Cuéllar JM, Lanman TH. "Text neck": an epidemic of the modern era of cell phones? Spine J 2017;17:901-2.

9. Choi JH, Jung MO, Yoo KT. An analysis of the activity and muscle fatigue of the muscles around the neck under the three most frequent postures while using a smartphone. J Phys Ther Sci 2016;28:1660-4.

10. Gustafsson E, Thomée S, Grimby-Ekman A, et al. Texting on mobile phones and musculoskeletal disorders in young adults: A five-year cohort study. Appl Ergon 2017;58:208-14.

11. Gustafsson E, Johnson PW, Lindegård A, et al. Technique, muscle activity and kinematic differences in young adults texting on mobile phones. (PMID) Ergonomics 2011;54:477-87.

12. Peper E, Harvey R. Digital addiction: increased loneliness, anxiety, and depression. Neuroregolation 2018;5:3-8.

13. De Pasquale C, Sciacca F, Hichy Z. Smartphone addiction and dissociative experience: an investigation in Italian adolescent aged between 14 and 19 years. International Journal of Psychology \& Behavior Analysis 2015;1:109.

14. Elhai JD, Dvorak RD, Levine JC, et al. Problematic smartphone use: conceptual overview and systematic review of relations with anxiety and depression psychopathology. J Affect Disord 2017;207:251-9.

15. Kwon M, Kim DJ, Cho H, et al. The smartphone addiction scale: development and validation of a short version for adolescents. PloS One 2013;8:e83558.

16. Lopez-Fernandez O. Short version of the smartphone addiction scale adapted to Spanish and French: towards a cross-cultural research in problematic mobile phone use. Addict Behav 2017;64:275-80.

17. De Pasquale C, Sciacca F, Hichy Z. Italian validation of 
smartphone addiction scale short version for adolescents and young adults (SAS-SV). Psichology 2017;8:1513-8

18. Giansanti D. Investigation of fall-risk using a wearable device with accelerometers and rate gyroscopes. Physiol Meas 2006;27:1081-90.

19. Giansanti D, Maccioni G. Comparison of three different kinematic sensor assemblies for locomotion study. Physiol Meas 2005;26:689-705.

20. Giansanti D, Maccioni G, Macellari V. The development and test of a device for the reconstruction of 3-D position and orientation by means of a kinematic sensor assembly with rate gyroscopes and accelerometers. IEEE Trans Biomed Eng 2005;52:1271-7.

21. Giansanti D. Introduction of medical Apps in telemedicine and e-health: problems and opportunities. Telemed J E Health 2017;23:773-6.

22. Censi F, Mattei E, Triventi M, et al. Regulatory

doi: $10.21037 /$ mhealth-20-150

Cite this article as: Giansanti D, Maccioni G. Health in the palm of your hand-part 1: the risks from smartphone abuse and the role of telemedicine and e-Health. mHealth 2021;7:49. frameworks for mobile medical applications. Expert Rev Med Devices 2015;12:273-8.

23. Baldani G. Regolamentare l'infinito: La sfida della Food and Drug Administration [Regulating infinity: The challenge of Food and Drug Administration. Health and Society]. Salute e Società 2014:171-5.

24. Salinetti S, De Castro P, Barbaro MC, et al. Alternanza scuola lavoro in ISS. Riflessioni a tre anni di attività [School-work alternation in ISS. Reflections on three years of activity, Newsletter Bullettin of the Istituto Superiore di Sanità]. Notiziario dell'Istituto Superiore di Sanità 2018;31:3-7.

25. Rossi AM, Barbaro MC, Salinetti S, et al. La notte europea dei ricercatori: un successo in crescita [European researchers' night: a growing success, Newsletter Bullettin of the Istituto Superiore di Sanità]. Notiziario dell'Istituto Superiore di Sanità 2018;31:13-6. 